\title{
Cost-Effectiveness of Nivolumab-Ipilimumab Combination Therapy Compared with Monotherapy for First-Line Treatment of Metastatic Melanoma in the United States
}

\author{
Anna Oh, MSN, MPH, RN; Dang M. Tran, BS; Leann C. McDowell, PharmD; Dor Keyvani, PharmD; \\ Jay Andrew Barcelon, BS; Oscar Merino, BS; and Leslie Wilson, PhD
}

\begin{abstract}
BACKGROUND: The approval of new immunotherapies has dramatically changed the treatment landscape of metastatic melanoma. These survival gains come with trade-offs in side effects and costs, as well as important considerations for third-party payer systems, physicians, and patients.
\end{abstract}

OBJECTIVE: To develop a Markov model to determine the cost-effectiveness of nivolumab, ipilimumab, and nivolumab-ipilimumab combination as firstline therapy in metastatic melanoma, while accounting for differential effectiveness in programmed death-ligand 1 (PD-L1) positive and negative patients.

METHODS: A 3-state Markov model (PD-L1 positive stable disease, PD-L1 negative stable disease, and progression and/or death) was developed using a U.S. societal perspective with a lifetime time horizon of 14.5 years. Transition probabilities were calculated from progression-free (PF) survival data reported in the CheckMate-067 trial. Costs were expressed in 2015 U.S. dollars and were determined using national sources. Adverse event $(A E)$ management was determined using immune-related $A E$ (irAE) data from CheckMate-067, irAE management guides for nivolumab and ipilimumab, and treatment guidelines. Utilities were obtained from published literature, using melanoma-specific studies when available, and were weighted based on incidence and duration of irAEs. Base case, one-way sensitivity, and probabilistic sensitivity analyses were conducted.

RESULTS: Nivolumab-ipilimumab combination therapy was not the costeffective choice (\$454,092 per PF quality-adjusted life-year [QALY]) compared with nivolumab monotherapy in a base case analysis at a willingness-to-pay threshold of $\$ 100,000$ per PFQALY. Combination therapy and nivolumab monotherapy were cost-effective choices compared with ipilimumab monotherapy. PD-L1 positive status, utility of nivolumab and combination therapy, and medication costs contributed the most uncertainty to the model. In a population of $100 \%$ PD-L1 negative patients, nivolumab was still the optimal treatment, but combination therapy had an improved incremental cost-effectiveness ratio (ICER) of $\$ 295,903$ per PFQALY. Combination therapy became dominated by nivolumab, when $68 \%$ of the sample was PD-L1 positive. In addition, the cost of ipilimumab would have to decrease to $<\$ 21,555$ per dose for combination therapy to have an ICER $<\$ 100,000$ per PFQALY and to $<\$ 19,151$ (a $42 \%$ reduction) to be more cost-effective than nivolumab monotherapy.

CONCLUSIONS: Nivolumab-ipilimumab combination therapy was not costeffective compared with nivolumab monotherapy, which was the most cost-effective option. Professionals in managed care settings should consider the pharmacoeconomic implications of these new immunotherapies as they make value-based formulary decisions, and future cost-effectiveness studies are completed.

J Manag Care Spec Pharm. 2017;23(6):653-64

Copyright $\odot 2017$, Academy of Managed Care Pharmacy. All rights reserved.

\section{What is already known about this subject}

The U.S. Food and Drug Administration recently approved nivolumab in combination with ipilimumab and nivolumab monotherapy as first-line treatment options to treat unresectable or metastatic melanoma.

The gains in response rates and survival come with trade-offs in side effects and costs, which are important considerations to third-party payer systems when determining the value of new, innovative therapies.

\section{What this study adds}

In the base case analysis, ipilimumab monotherapy was dominated by nivolumab monotherapy, while nivolumab-ipilimumab combination therapy had an incremental cost-effectiveness ratio (ICER) of $\$ 454,092$ per progression-free quality-adjusted lifeyear (PFQALY) gained compared with nivolumab.

Programmed death-ligand 1 positive status, utility of nivolumab and combination therapy, and medication costs contributed the most uncertainty to the model; these 3 factors are important to consider when determining the pharmacoeconomic implications of nivolumab monotherapy, ipilimumab monotherapy, and combination therapy.

At a willingness-to-pay threshold of $\$ 100,000$ per PFQALY, nivolumab monotherapy is the most cost-effective option compared with combination therapy and ipilimumab monotherapy.

工 n the United States, skin cancer is the most commonly diagnosed cancer, with more than 2 million people diagnosed annually. In 2015, almost 74,000 new cases were a result of melanoma, the most serious type of skin cancer that results in the most deaths. ${ }^{1-3}$ The median age at diagnosis is 63 years, and the long-term survival rate is less than $10 \%$ for stage IV metastatic melanoma., With an increasing elderly population and the development of costly treatments, the estimated cost of health care treatment for melanoma in the United States was $\$ 2.8$ billion in 2016, and this number is projected to increase. ${ }^{5}$

Before 2011, only 2 systemic therapies, dacarbazine and high-dose interleukin-2 (HD IL-2), were approved by the U.S. Food and Drug Administration (FDA) to treat 
metastatic melanoma, but neither treatment showed any benefit in overall survival and caused severe dose-limiting toxicities. ${ }^{6}$ Beginning in 2011, the treatment landscape for metastatic melanoma changed with the approval of novel immunotherapies such as ipilimumab (Yervoy) and targeted therapies such as vemurafenib (Zelboraf), dabrafenib (Tafinlar), and trametinib (Mekinist), all of which improved overall survival benefit. ${ }^{6-11}$ In 2014, two additional immunotherapies, pembrolizumab (Keytruda) and nivolumab (Opdivo) were approved for the treatment of unresectable or metastatic melanoma. Both treatments were associated with improvements in progressionfree survival (PFS), overall survival (OS), and overall objective responses. ${ }^{12-14}$

In response to these FDA approvals, the National Comprehensive Cancer Network (NCCN) Clinical Practice Guidelines in Oncology (NCCN Guidelines) revised the melanoma treatment recommendations (version 3.2015) to include HD IL-2, nivolumab, ipilimumab, and pembrolizumab as first-line treatment options for patients with metastatic or unresectable melanoma, with anticipated clinical stability of at least 12 weeks for BRAF V600 mutant and wild types. ${ }^{15}$ While nivolumab and ipilimumab were given category 1 recommendations based on uniform consensus and high-level evidence, no single treatment option was identified as the superior choice for first-line treatment. ${ }^{4}$

In 2015, the FDA granted accelerated approval for the use of nivolumab in combination with ipilimumab (combination therapy) to treat unresectable or metastatic melanoma because of significant increases in objective response rate $(60 \%)$ and PFS (median 11.5 months compared with median of 5 months for pembrolizumab and nivolumab monotherapies) in phase II and III clinical trials, giving clinicians another first-line treatment option. ${ }^{12,16,17}$ This combination therapy was included in version 2.2016 of the NCCN Guidelines as another first-line option. ${ }^{18}$ Ipilimumab monotherapy was demoted to a secondline treatment or subsequent therapy option after the use of nivolumab, pembrolizumab, or combination therapy in version 3.2016 of the NCCN Guidelines. ${ }^{19}$

The gains in response rates and survival come with tradeoffs in adverse events (AEs) and costs, which are important considerations for third-party payer systems when determining the value of these new, innovative therapies. In 2015, for a single dose of immunotherapy, the average wholesale price (AWP) from RED BOOK for a $70 \mathrm{~kg}$ patient was $\$ 5,732$ for nivolumab, $\$ 33,162$ for ipilimumab, and $\$ 35,073$ for combination therapy. AEs from these immunotherapies include immune-related reactions such as diarrhea, colitis, rash, endocrine disorders, hepatotoxicity, and pneumonitis. ${ }^{20}$ Grade 3 or 4 AEs requiring hospitalization or urgent treatment are somewhat common, as $55 \%$ of patients in the combination group, $20 \%-27 \%$ of patients in the ipilimumab monotherapy group, and 16\% of patients in the nivolumab monotherapy group experienced these treatment-related AEs. ${ }^{12,14,17}$ These are important considerations for oncologists and patients. since treatment response, costs (drug and AE management), and incidence of AEs may affect treatment decisions and patient quality of life. Therefore, it is uncertain whether one treatment provides true incremental cost-effectiveness value over the others in terms of efficacy, toxicity, and cost.

The Academy of Managed Care Pharmacy (AMCP) has its own guidelines called the AMCP Format for Formulary Submission, which urges health plans to request a formal dossier from drug companies on a new drug's effectiveness and safety and its economic value relative to alternative therapies to inform value-based decisions in formulary management. ${ }^{21,22}$ However, health care decision makers have criticized the dossiers submitted by manufacturers as biased. ${ }^{22}$ Two separate analyses have concluded that combination therapy and nivolumab monotherapy were respectively cost-effective compared with ipilimumab in an Australian population. ${ }^{23,24}$ Both analyses were published as abstracts by industry authors based in Australia.

Our cost-effectiveness study comparing combination therapy to nivolumab and ipilimumab monotherapies in 1 analysis is meant to add value-based evidence without having ties to industry, which better informs decision makers when assessing the value of new drugs by AMCP formulary guidelines. Also, no analysis has been performed in the United States or has incorporated programmed death-ligand 1 (PD-L1) status when comparing the 3 treatments. These are notable differences because of the potential use of targeting treatment to the PD-Ll status of patients, which is an emerging consideration, since multiple subgroup analyses from clinical trials for PD-1 inhibitors suggest that the greatest benefit occurs in patients with PD-L1 positive tumors. ${ }^{25}$ When drugs are better targeted to those that benefit, they are also more cost-effective. In addition, the cost of the drugs and the willingness-to-pay (WTP) threshold varies by country, so performing an analysis in the United States could lead to changes in the results and subsequent treatment decisions by U.S. decision makers. ${ }^{26}$

The purpose of this study, then, was to determine the costeffectiveness of nivolumab-ipilimumab combination therapy compared with nivolumab and ipilimumab monotherapies as first-line treatments for patients with confirmed stage III or IV melanoma from a U.S. societal perspective. Managed care professionals can use these findings to better understand the comparative value of these treatments with respect to efficacy, toxicity, and cost and make informed value-based formulary decisions on the role of each drug regimen in the treatment of melanoma.

\section{Methods}

We performed a cost-effectiveness analysis (CEA), which compared 2 or more alternative treatments to determine which one provided greater benefit at the same or lower costs, lower costs 
Cost-Effectiveness of Nivolumab-Ipilimumab Combination Therapy Compared

with Monotherapy for First-Line Treatment of Metastatic Melanoma in the United States

\section{FIGURE 1 Markov Model: Abbreviated Decision Tree Comparing Therapies and Schematic Representation}

A. Abbreviated Markov model decision tree comparing nivolumab-ipilimumab combination therapy, nivolumab monotherapy, and ipilimumab monotherapy for first-line therapy for metastatic melanoma while incorporating PD-L1 status

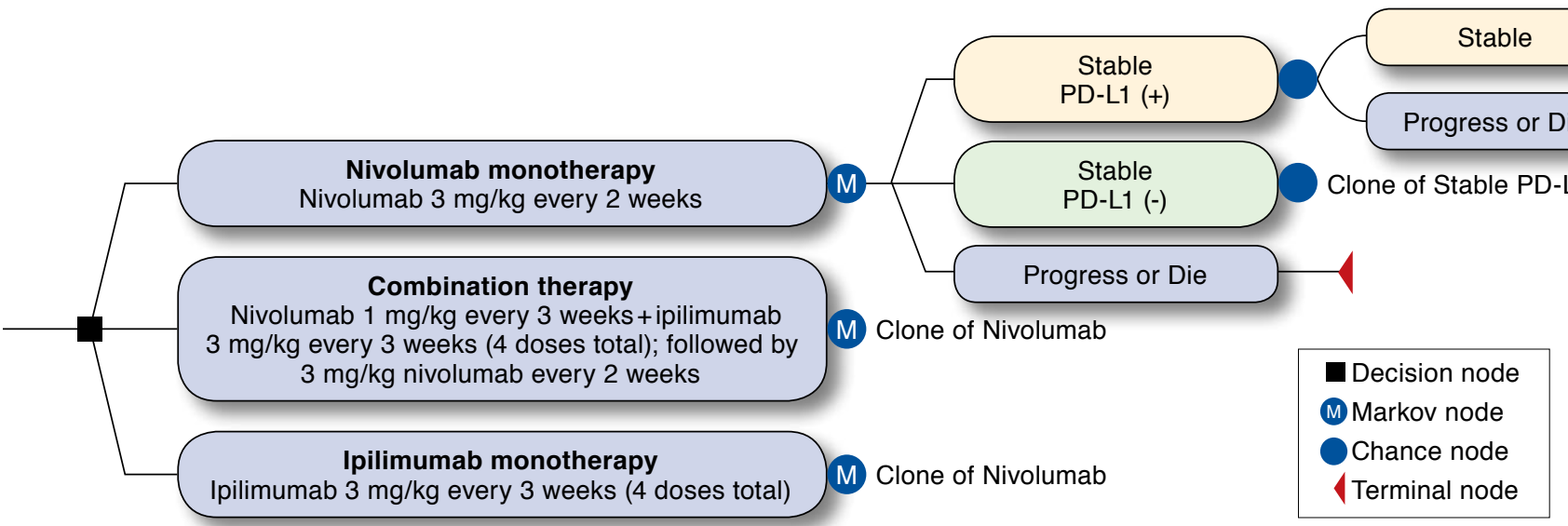

\section{B. Schematic representation of the Markov model showing disease states and transitions}

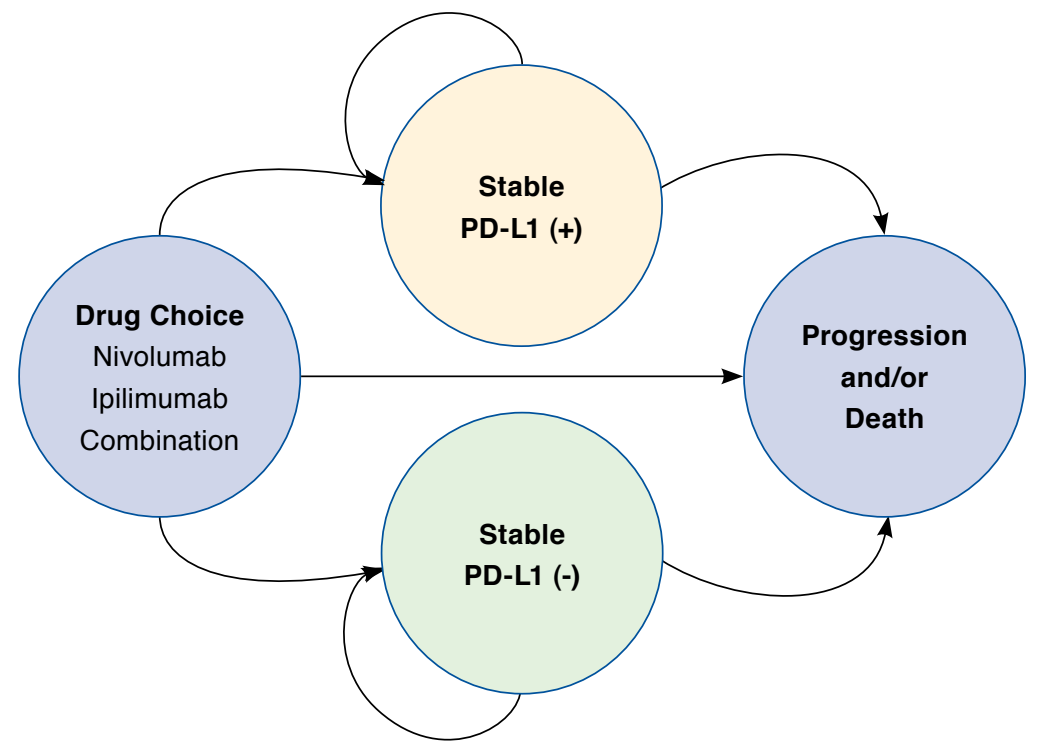

PD-L1 = programmed death-ligand 1 .

for the same or greater benefit, or greater benefit and lower costs. ${ }^{27}$ CEA results were expressed as the incremental costeffectiveness ratio (ICER) in U.S. dollars per quality-adjusted life-years (QALYs) saved. If the ICER was less than a WTP threshold (commonly $\$ 100,000$ in the United States), ${ }^{28}$ then it was considered cost-effective to adopt that treatment over the alternative. $^{27,29}$

As with our study, most CEA studies are Markov models based on efficacy outcomes from clinical trial literature and other published data to assess the cost and benefit of each treatment and its side effects. ${ }^{29}$ Our model included 7 steps:
(1) define the study population, (2) define the health states of the Markov model as patients moved from treatments to death, (3) determine the costs of moving from one health state to another, (4) determine the probability of patients transitioning from one health state to another, (5) calculate the ICER for each comparison for the base-case of assumptions, (6) determine which treatment is the most cost-effective (has the lowest ICER compared with the next costly alternative and under the $\$ 100,000$ WTP threshold), and (7) conduct sensitivity analysis on all factors of the model to see which factors change the CEA decision. $^{27,29}$ Our methods adhered to the recommendations 
Cost-Effectiveness of Nivolumab-Ipilimumab Combination Therapy Compared with Monotherapy for First-Line Treatment of Metastatic Melanoma in the United States

TABLE 1 Model Input Parameters, Distribution, and Range

\begin{tabular}{|c|c|c|c|c|}
\hline \multirow[b]{2}{*}{ Parameter } & \multirow[b]{2}{*}{ Base Case } & \multicolumn{2}{|c|}{ Range } & \multirow[b]{2}{*}{ Source } \\
\hline & & Low & High & \\
\hline \multicolumn{5}{|l|}{ Drug costs $^{\mathrm{a}}(\$ ; \gamma$ distribution $)$} \\
\hline Nivolumab & 85,983 & 64,487 & 107,478 & \multirow{3}{*}{ RED BOOK, $\pm 25 \%$} \\
\hline Ipilimumab & 132,649 & 99,487 & 165,811 & \\
\hline Combination therapy: nivolumab; ipilimumab & 7,$643 ; 132,649$ & 5,$732 ; 99,487$ & 9,$554 ; 165,811$ & \\
\hline \multicolumn{5}{|l|}{ Total treatment-associated costs ${ }^{\mathrm{b}}(\$ ; \gamma$ distribution $)$} \\
\hline Nivolumab & 12,036 & 9,027 & 15,045 & \multirow{3}{*}{$\begin{array}{c}\text { Medical Fee Book 2015, } \\
\text { CLFS 2015 } \\
\pm 25 \%\end{array}$} \\
\hline Ipilimumab & 5,219 & 3,914 & 6,524 & \\
\hline Combination therapy: nivolumab; ipilimumab & 5,$219 ; 253$ & 3,$914 ; 190$ & 6,$524 ; 316$ & \\
\hline \multicolumn{5}{|l|}{ Cost of managing adverse events $(\$ ; \gamma$ distribution $)$} \\
\hline Nivolumab & 2,688 & 2,015 & 3,359 & \multirow{3}{*}{ HCUP, $\pm 25 \%$} \\
\hline Ipilimumab & 7,049 & 5,286 & 8,811 & \\
\hline Combination therapy: nivolumab; ipilimumab & 14,415 & 10,811 & 18,019 & \\
\hline Cost of disease progression/death $(\$ ; \gamma$ distribution) & 68,849 & 34,424 & 137,697 & Guy et al., $2012^{48}$ \\
\hline \multicolumn{5}{|l|}{ Utilities $^{\mathrm{c}}(\gamma$ distribution $)$} \\
\hline Stable disease, base case & 0.667 & 0.500 & 0.833 & \multirow{8}{*}{$\begin{array}{c}\text { Beusterien et al., } 200937 \\
\text { Lloyd et al., } 2006^{38} \\
\text { Swinburn et al., } 2012^{39} \\
\text { Dobler et al., 201540 } \\
\text { Chen et al., 200441 } \\
\text { Nolan et al., } 1985^{42} \\
\pm 25 \%\end{array}$} \\
\hline Progressive disease, base case & 0.433 & 0.325 & 0.542 & \\
\hline Nivolumab stable & 0.131 & 0.098 & 0.164 & \\
\hline Nivolumab progression/death & 0.084 & 0.063 & 0.106 & \\
\hline Ipilimumab stable & 0.129 & 0.097 & 0.162 & \\
\hline Ipilimumab progression/death & 0.082 & 0.062 & 0.104 & \\
\hline Combination stable & 0.119 & 0.089 & 0.149 & \\
\hline Combination progression/death & 0.072 & 0.054 & 0.091 & \\
\hline \multicolumn{5}{|c|}{$\begin{array}{l}{ }^{a} \text { Cost for complete course corresponds to median number of doses. All drug prices are average wholesale price minus } 17 \% \text {. } \\
\text { bIncludes cost of outpatient initial visit, follow-up visits, lab draws, and follow-up imaging as recommended by the manufacturer of nivolumab and ipilimumab, treatment } \\
\text { guidelines, and expert opinion; total cost is based on number of procedures for each visit and median number of doses for each treatment. } \\
\text { cUtilities from the literature were based on standard gamble and time trade-off; utility values are listed after weighting 10/12 for stable and progression and } 2 / 12 \text { for } \\
\text { treatment with irAEs. } \\
\text { CLFS= Clinical Laboratory Fee Schedule; HCUP = Healthcare Cost and Utilization Project; irAE=immune-related adverse event. }\end{array}$} \\
\hline
\end{tabular}

of good research practices for model transparency and validation from the International Society for Pharmacoeconomics and Outcomes Research (ISPOR), Society of Medical Decision Making, and the Consolidated Health Economic Evaluation Reporting Standards (CHEERS) statement from the ISPOR Task Force. ${ }^{27,30,31}$

\section{Study Population}

The decision tree and Markov model for our CEA were based on the phase III, randomized controlled trial CheckMate-067, for first-line treatment of confirmed stage III or IV unresectable or metastatic melanoma. ${ }^{12}$ Eligible patients were aged at least 18 years and had an Eastern Cooperative Oncology Group (ECOG) performance-status (PS) score of 0 or 1 , measurable disease as assessed by computed tomography or magnetic resonance imaging, known PD-Ll status, and known BRAF V600 mutation status. There were no significant between-group differences at baseline. The mean age was 60 years for all patients (59 in both nivolumab and combination arms and 61 in the ipilimumab arm). Males made up $64 \%$ of the total sample; $73 \%$ had an ECOG PS of 0; 32\% were positive for the BRAF V600 mutation; and $24 \%$ had positive PD-L1 status. ${ }^{12}$
Treatment options in our model reflected those in the CheckMate-067 clinical trial: (a) combination therapy (nivolumab $1 \mathrm{mg}$ per $\mathrm{kg}[\mathrm{mg} / \mathrm{kg}$ ] plus ipilimumab $3 \mathrm{mg} / \mathrm{kg}$ every 3 weeks for 4 doses, followed by nivolumab $3 \mathrm{mg} / \mathrm{kg}$ every 2 weeks); (b) nivolumab $3 \mathrm{mg} / \mathrm{kg}$ every 2 weeks; and (c) ipilimumab $3 \mathrm{mg} / \mathrm{kg}$ every 3 weeks for a total of 4 doses. The median number of doses was 4 for combination therapy, 15 for nivolumab, and 4 for ipilimumab. ${ }^{12}$ Treatment was discontinued if the disease progressed, unacceptable AEs developed, or patients withdrew consent.

\section{Markov Model}

The Markov model was developed using TreeAge Pro (TreeAge, Williamstown, MA) with 3 branches at the decision node to reflect the 3 drug treatments being compared: combination therapy, nivolumab monotherapy, and ipilimumab monotherapy (Figure 1A). The cycle length for the model was 1 month, and the time horizon was 175 months, which is consistent with the 10-year mortality rate of metastatic melanoma. ${ }^{1}$ The health states after a patient received 1 of the treatment options was "stable disease with positive PD-L1 status," "stable disease with 
Cost-Effectiveness of Nivolumab-Ipilimumab Combination Therapy Compared with Monotherapy for First-Line Treatment of Metastatic Melanoma in the United States

TABLE 2 Proportion of Patients from CheckMate-067 Experiencing irAEs and Utility Estimate by Organ System

\begin{tabular}{|c|c|c|c|c|c|c|c|}
\hline \multirow[b]{2}{*}{ Patients Experiencing irAEs, \% } & \multicolumn{2}{|c|}{ Nivo $(\mathbf{n}=313)$} & \multicolumn{2}{|c|}{ Combo $(n=313)$} & \multicolumn{2}{|c|}{ Ipi $(n=311)$} & \multirow[b]{2}{*}{ Utility ${ }^{38-42}$} \\
\hline & Grade 3/4 & Grade $1 / 2$ & Grade $3 / 4$ & Grade $1 / 2$ & Grade $3 / 4$ & Grade $1 / 2$ & \\
\hline Skin & 1.6 & 40.3 & 5.8 & 53.4 & 2.9 & 51.1 & \multirow{5}{*}{-0.057} \\
\hline Pruritis & 0.0 & 18.8 & 1.9 & 31.3 & 0.3 & 35.0 & \\
\hline Rash & 0.3 & 21.4 & 2.9 & 25.6 & 1.6 & 19.3 & \\
\hline Rash maculo-papular & 0.3 & 3.8 & 1.9 & 9.9 & 0.3 & 11.6 & \\
\hline Vitiligo & 0.3 & 7.0 & 0.0 & 6.7 & 0.0 & 3.9 & \\
\hline Gastrointestinal & 2.2 & 17.3 & 14.7 & 31.6 & 11.6 & 25.1 & \multirow{3}{*}{-0.116} \\
\hline Diarrhea & 2.2 & 16.9 & 9.3 & 34.8 & 6.1 & 27.0 & \\
\hline Colitis & 0.6 & 0.6 & 7.7 & 4.2 & 8.7 & 2.9 & \\
\hline Hepatic & 2.6 & 3.8 & 18.8 & 11.2 & 1.6 & 5.5 & \multirow{3}{*}{-0.308} \\
\hline Increase in ALT & 1.3 & 2.6 & 8.3 & 9.3 & 1.6 & 2.3 & \\
\hline Increase in AST & 1.0 & 2.9 & 6.1 & 9.3 & 0.6 & 2.9 & \\
\hline Endocrine & 0.6 & 13.7 & 4.8 & 25.2 & 2.3 & 8.7 & \multirow{4}{*}{-0.115} \\
\hline Hypothyroidism & 0.0 & 8.6 & 0.3 & 14.7 & 0.0 & 4.2 & \\
\hline Hyperthyroidism & 0.0 & 4.2 & 1.0 & 8.9 & 0.0 & 1.0 & \\
\hline Hypophysitis & 0.3 & 0.3 & 1.6 & 6.1 & 1.9 & 1.9 & \\
\hline Pulmonary & 0.3 & 1.3 & 1.0 & 6.1 & 0.3 & 1.6 & \multirow{2}{*}{-0.159} \\
\hline Pneumonitis & 0.3 & 1.0 & 1.0 & 5.4 & 0.3 & 1.3 & \\
\hline
\end{tabular}

negativePD-L1 status," and "progressionand/ordeath"(Figure 1B). The model's population started in the stable health state, separated by PD-Ll status based on the proportion of positive PD-L1 patients from CheckMate-067. In each simulation cycle, patients either stayed in the stable state or advanced to the terminal node of progression/death. The simulation continued until all patients entered the progression/death state.

For the base case analysis, we assessed effectiveness as months of PFS, and the ICER was expressed as U.S. dollars per progression-free quality-adjusted life-year (PFQALY). Estimated treatment costs from our model results were ranked from lowest to highest. We assumed treatments were cost-effective based on a WTP threshold of $\$ 100,000$ per PFQALY gained.

Acceptability curves are a method for summarizing information on uncertainty around a cost-effectiveness ICER. ${ }^{27,29}$ These curves show the probability that an intervention is costeffective compared with an alternative for a range of maximum thresholds that a decision maker would be willing to pay for a particular unit change in outcome. One-way and probabilistic sensitivity analyses (PSAs) were used to examine the uncertainties surrounding the model's assumptions, including characteristics of baseline population PD-Ll status, treatment efficacy, utilities, costs, and uncertainty in other model parameters (Table 1). PSA was performed with 10,000 Monte Carlo simulations to simultaneously account for uncertainty in all utility and cost parameters, including transition probabilities at each cycle (Table 1). ${ }^{32}$ We assigned beta distributions to probability and utility estimates, since both are bounded by zero at the lower end and 1 at the upper end. ${ }^{29}$ Zero represents the worst possible health state, and 1 represents perfect health in the utility estimates. For cost estimates, we assigned gamma distributions, since cost data are often highly skewed and represented by infinity at the upper end. ${ }^{29}$

\section{Transition Probabilities}

Transition probabilities represent the proportion of the population that would move from one health state into another health state after 1 cycle. ${ }^{29}$ For example, if 1 patient from a population of 100 patients in the stable PD-Ll state moved into the progression and/or death state after 1 cycle, then the transition probability would be 0.01 . To calculate monthly transition probabilities, PFS data for all 3 treatments, separated by PD-L1 status, were extracted from the published Kaplan-Meier curves in the CheckMate-067 clinical trial, using a validated graphical digitizer (WebPlotDigitizer, version 3.9; Ankit Rohatgi, Austin, TX; Appendix A, available in online article). ${ }^{12}$ The extracted data were used to calculate the survival function, hazard function, and transition probabilities. We assumed patients who survived through the end of the clinical trial had longer survival and might be different than those who had died from their malignancy, since the tail of the Kaplan-Meier curve from Checkmate-067 appeared to level off. ${ }^{12}$ In order to preserve the treatment effect and extrapolate the tail of Kaplan-Meier curves to zero, we carried forward the last observed transition probability rather than using the Declining Exponential Approximation of Life Expectancy method, which overestimates mortality rate in the tails. ${ }^{33} \mathrm{PFS}$ data were used, since OS data had not been published at the time of this analysis. 
TABLE 3 Summary of the Cost-Effectiveness of Nivolumab, Ipilimumab, and Combination as First-Line Treatment of Metastatic Melanoma and One-Way Deterministic Sensitivity Analysis from the Markov Model

\begin{tabular}{|c|c|c|c|c|c|}
\hline Treatment & $\begin{array}{l}\text { Total Cost } \\
(95 \% \text { CI; \$) }\end{array}$ & $\begin{array}{c}\text { Total Effectiveness } \\
\text { PFQALY } \\
\text { (95\% CI) }\end{array}$ & $\begin{array}{c}\text { Incremental Cost } \\
(95 \% \mathrm{CI} ; \$)\end{array}$ & \begin{tabular}{|c|} 
Incremental Effect \\
PFQALY \\
$(95 \%$ CI $)$
\end{tabular} & $\begin{array}{c}\text { ICER per PFQALY } \\
(\$)\end{array}$ \\
\hline \multicolumn{6}{|c|}{ Base case analysis (PD-L1 status unknown) } \\
\hline Nivolumab & 169,320 & 4.24 & - & - & - \\
\hline Ipilimumab & 213,763 & 3.68 & 44,443 & -0.57 & (dominated) \\
\hline Combo vs. ipilimumab & 228,352 & 4.37 & 14,589 & 0.69 & 21,143 \\
\hline Combo vs. nivolumab & 228,352 & 4.37 & 59,032 & 0.13 & 454,092 \\
\hline \multicolumn{6}{|c|}{ PD-L1 status known (100\% PD-L1 negative) } \\
\hline Nivolumab & 169,449 & 4.14 & - & - & - \\
\hline Ipilimumab & 213,765 & 3.62 & 44,316 & -0.52 & (dominated) \\
\hline Combo vs. ipilimumab & 228,387 & 4.34 & 14,622 & 0.72 & 20,308 \\
\hline Combo vs. nivolumab & 228,387 & 4.34 & 58,937 & 0.20 & 295,903 \\
\hline \multicolumn{6}{|c|}{ PD-L1 status known (100\% PD-L1 positive) } \\
\hline Nivolumab & 168,900 & 4.57 & - & - & - \\
\hline Ipilimumab & 213,757 & 3.86 & 44,857 & -0.72 & (dominated) \\
\hline Combo vs. ipilimumab & 228,241 & 4.46 & 14,484 & 0.60 & 24,140 \\
\hline Combo vs. nivolumab & 228,241 & 4.46 & 59,342 & -0.11 & (dominated) \\
\hline \multicolumn{6}{|c|}{ Utilities unadjusted for adverse events } \\
\hline Nivolumab & 169,320 & 4.26 & - & - & - \\
\hline Ipilimumab & 213,763 & 3.70 & 44,444 & -0.55 & (dominated) \\
\hline Combo vs. ipilimumab & 228,352 & 4.47 & 14,589 & 0.77 & 18,947 \\
\hline Combo vs. nivolumab & 228,352 & 4.47 & 59,033 & 0.21 & 275,544 \\
\hline \multicolumn{6}{|c|}{ Ipilimumab monotherapy $=\$ 73,520$} \\
\hline Ipilimumab & 154,636 & 3.68 & - & - & - \\
\hline Combo & 169,226 & 4.37 & 14,589 & 0.69 & 21,090 \\
\hline Nivolumab & 169,320 & 4.24 & 94.06 & -0.13 & (dominated) \\
\hline \multicolumn{6}{|c|}{ Ipilimumab monotherapy $=\$ 86,272$} \\
\hline Ipilimumab & 167,387 & 3.68 & - & - & - \\
\hline Nivolumab & 169,320 & 4.24 & 1,932 & 0.57 & 3,420 \\
\hline Combo & 181,976 & 4.37 & 12,657 & 0.13 & 99,872 \\
\hline \multicolumn{6}{|c|}{ Ipilimumab monotherapy $=\$ 88,171$} \\
\hline Ipilimumab & 169,286 & 3.68 & - & - & - \\
\hline Nivolumab & 169,320 & 4.24 & 33.27 & 0.57 & 58.88 \\
\hline Combo & 183,876 & 4.37 & 14,556 & 0.13 & 114,858 \\
\hline \multicolumn{6}{|c|}{ Ipilimumab monotherapy $=\$ 88,443$} \\
\hline Nivolumab & 169,320 & 4.24 & - & - & - \\
\hline Ipilimumab & 169,558 & 3.68 & 238.03 & -0.57 & (dominated) \\
\hline Combo vs. ipilimumab & 184,147 & 4.37 & 14,589 & 0.69 & 21,143 \\
\hline Combo vs. nivolumab & 184,147 & 4.37 & 14,827 & 0.13 & 116,999 \\
\hline
\end{tabular}

aIn 2015 U.S. dollars.

$C I=$ confidence interval; ICER = incremental cost-effectiveness ratio; $P D-L 1$ = programmed death-ligand 1; PFQALY=progression-free quality-adjusted life-year.

\section{Costs and Utilities}

Costs of drug treatment, drug administration, and treatmentrelated AE management were applied up front as an initial cost, while costs associated with the progression/death health state were applied as a final cost. National registries were used to determine costs when available. All costs were adjusted to 2015 U.S. dollars, and drug costs were discounted at 3\% annually (Table 1). ${ }^{27,29}$ Drug costs were taken from RED BOOK Online 2015 as AWP in 2015 U.S. dollars and were discounted by $17 \%$ to account for contract pricing and to be consistent with estimates for Medicare reimbursement. ${ }^{34}$ The costs of drug treatment were calculated using a standard average U.S. patient weight of $74.4 \mathrm{~kg}$, rounded down to $70 \mathrm{~kg}$ for weight loss effects of the cancer stage ${ }^{35}$ and were weighted based on the dosing regimen and median duration of treatment from CheckMate-067. ${ }^{12}$

Treatment-associated costs were determined using Current Procedural Terminology codes, the 2015 Medical Fee Book, 
Cost-Effectiveness of Nivolumab-Ipilimumab Combination Therapy Compared with Monotherapy for First-Line Treatment of Metastatic Melanoma in the United States

FIGURE 2 Acceptability Curve and One-Way Sensitivity Analysis

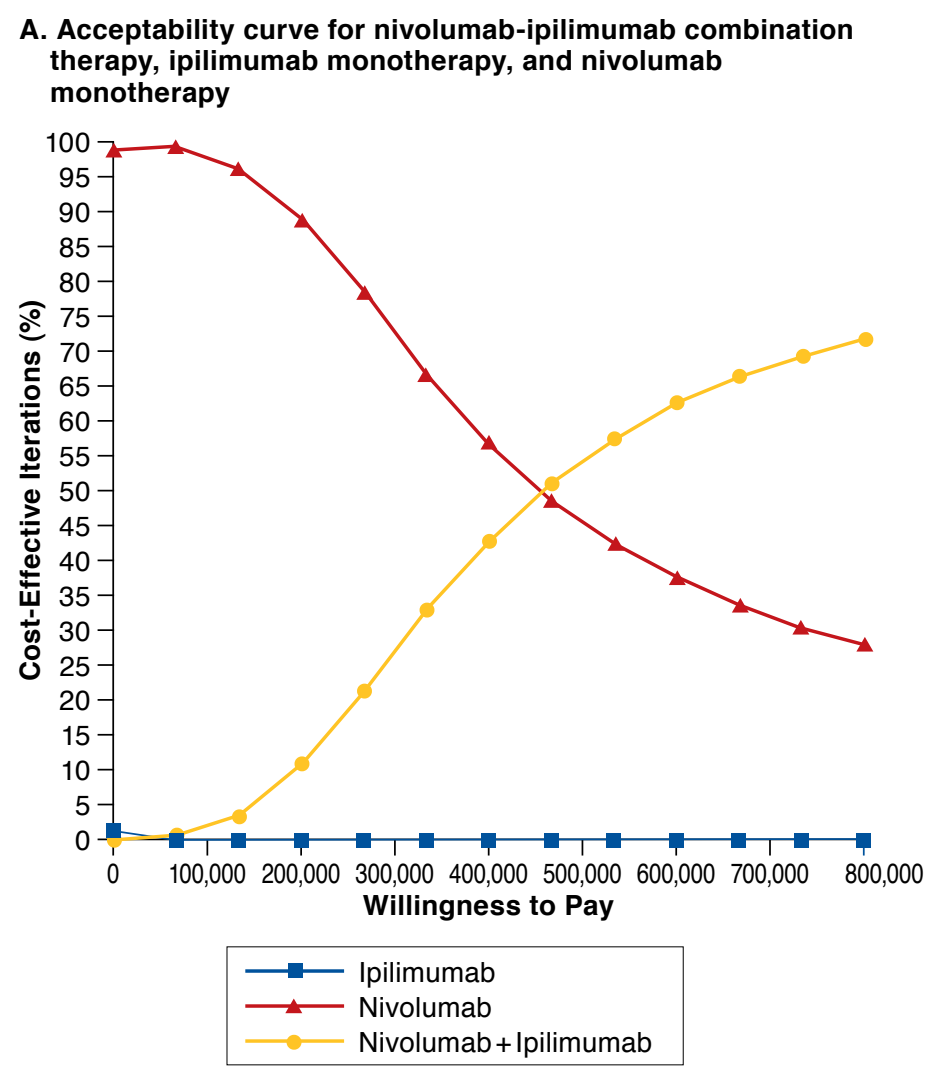
B. One-way sensitivity analysis for proportion of PD-L1 positive patients

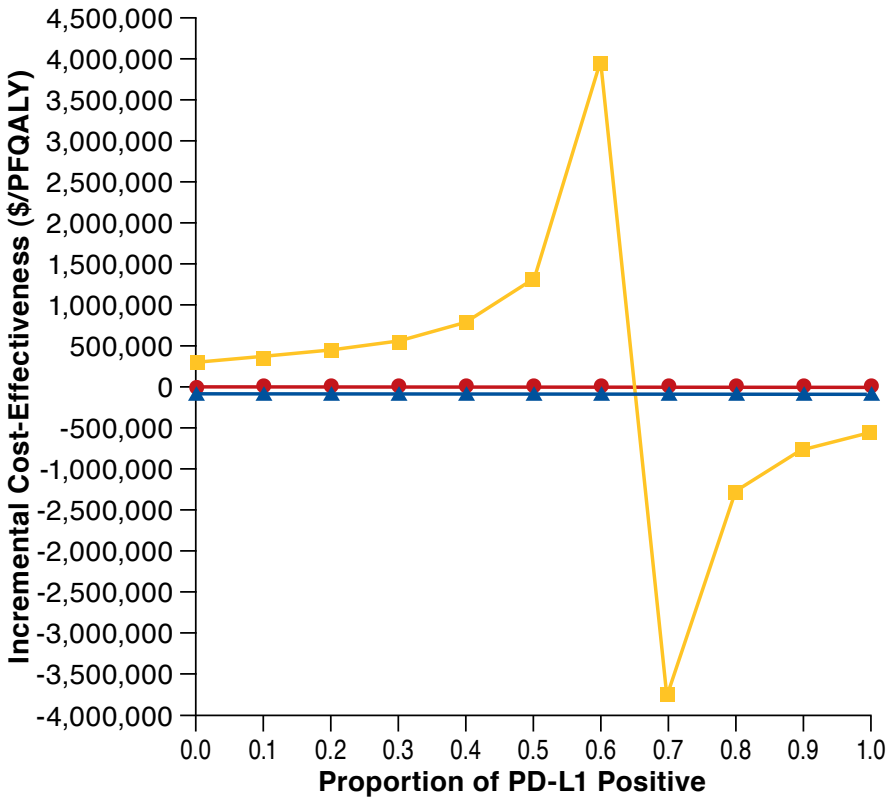

PD-L1 = programmed death-ligand 1; PFQALY= progression-free quality-adjusted life-year.

and the 2015 Clinical Laboratory Fee Schedule and included physician fees, outpatient office visits, laboratory tests, infusion costs, and imaging tests to monitor disease progression. Treatment-associated costs were calculated based on the recommended schedule from the study protocol and median duration of each treatment.

The probabilities for organ-specific AEs were taken from CheckMate-067, and grade 1 and 2 AEs were separated from grade 3 and 4 AEs (Table 2). All nonimmune-related AEs were excluded. We explicitly accounted for costs and utilities only associated with immune-related AEs (irAEs) reported by at least $5 \%$ of patients in any of the treatment groups, since these irAEs are most concerning to clinicians and most likely to cause severe episodes that result in costly hospitalizations. ${ }^{12}$ Costs and utilities for the management of irAEs were weighted based on the percentage of patients experiencing that irAE and the median time to resolution ( 2 months) for irAEs as reported in Checkmate-067.

Costs for irAE management included drugs used to treat irAEs, consultations needed while hospitalized, and laboratory and imaging tests used to diagnose and monitor the irAEs. Management of irAEs was determined using the study protocol, AE management guides supplied by the manufacturer, treatment guidelines, and expert clinical knowledge. ${ }^{12}$ The model assumed that all patients with an irAE that required steroid treatment would receive oral prednisone as per consensus management ${ }^{36}$ and with dosing based off of the average dose as listed and recommended in the manufacturer's AE management guide, a patient weight of $70 \mathrm{~kg}$, and the median time to resolution of the irAE. ${ }^{12}$ Per consensus management, prednisone tapers occur with a $10 \mathrm{mg}$ dose decrease every week until a $5 \mathrm{mg}$ dose is achieved.$^{36}$ The model also assumed that all grade 3 and 4 irAEs would incur a hospitalization charge, and once discharged from the hospital, patients would receive follow-up outpatient visits to monitor the irAE every 2 weeks. Grade 1 and 2 irAEs would not require hospitalization, so follow-up outpatient visits to monitor the irAE would occur monthly.

Baseline utility estimates for stable and progression/death health states and utility values for irAEs grouped by organ system were obtained from previously published health 
preferences studies based on patients with advanced melanoma or other malignancies. ${ }^{37-42}$ Methodologies for these health preference studies included standard gamble and time tradeoff. Utilities for stable and progression/death health states for each treatment arm were calculated as an annual (12 months) weighted utility. Thus, the weighted utility values for irAEs were multiplied by $2 / 12$ (median time of 2 months to resolution) and then subtracted from the baseline utility value of stable and progressive disease that was multiplied by $10 / 12$ (remaining 10 months without the irAE). The use of weighted utility values allowed for more accurate estimations of the utilities given the varying time frame that a patient may experience an irAE. The utility value for the progression/death health state was divided in half to account for the utility during the time in the progressed state and during the time in a dead state when the utility would be zero. Utility values were applied upfront in the model at the decision node before initiation of nivolumab monotherapy, ipilimumab monotherapy, or combination therapy (Figure 1A).

\section{Results}

\section{Cost-Effectiveness Analysis}

In the base case analysis, which represents our best available estimates, nivolumab monotherapy had the lowest overall cost at $\$ 169,320$, followed by ipilimumab monotherapy at $\$ 213,763$ and combination therapy, which was the most expensive at $\$ 228,352$ (Table 3). Combination therapy provided an additional 0.69 PFQALYs with an incremental cost of $\$ 14,589$, compared with ipilimumab, and an additional 0.13 PFQALYs with an incremental cost of $\$ 59,032$, compared with nivolumab. The model demonstrated that nivolumab was less expensive and more effective than ipilimumab, so nivolumab dominated and was the cost-effective choice compared with ipilimumab.

Combination therapy was not cost-effective when compared with nivolumab at a WTP threshold of $\$ 100,000$ per PFQALY because its ICER of $\$ 454,092$ per PFQALY gained was greater than the WTP threshold. However, combination therapy was cost-effective compared with ipilimumab at the same WTP threshold, with an ICER of $\$ 21,143$ per PFQALY gained. The drivers of this difference were the higher costs and shorter PFS time of ipilimumab compared with nivolumab.

Based on our base-case analysis, it would be cost-effective to choose nivolumab over ipilimumab for monotherapy decisions alone. However, combination therapy is not a cost-effective choice compared with nivolumab (the least costly option), but combination therapy is cost-effective compared with ipilimumab (the costlier monotherapy). Therefore, combination therapy can remain a cost-effective option in some cases.

\section{Sensitivity Analyses}

The acceptability curve (Figure 2A) confirmed the findings from our base case analysis with nivolumab as the optimal treatment below a WTP threshold of approximately $\$ 450,000$ per PFQALY. With increasing WTP thresholds, the probability that combination therapy was the optimal treatment increased, whereas the probability for nivolumab as optimal treatment decreased. Ipilimumab was not a viable treatment option for any WTP threshold between zero and $\$ 800,000$, since it was dominated by nivolumab and combination therapy throughout this range.

The tornado analysis (Appendix B, available in online article) showed that $\mathrm{PD}-\mathrm{Ll}$ positive status, utility of nivolumab in progressive disease adjusted for irAEs, and utility of combination therapy in progression adjusted for irAEs contributed the most uncertainty to our model, justifying additional exploratory one-way and probabilistic sensitivity analyses to identify how our decision would change if all patients were assumed PD-Ll positive or negative in the model. In the oneway sensitivity analysis that examined a population of $100 \%$ PD-L1 negative patients in all treatment groups, nivolumab was still the optimal treatment, since combination therapy had an ICER of $\$ 295,903$ per PFQALY, exceeding the WTP threshold of $\$ 100,000$ per PFQALY (Table 3). When the entire population was $100 \% \mathrm{PD}-\mathrm{Ll}$ positive, combination therapy and ipilimumab were dominated by nivolumab, further supporting the baseline case findings. Combination therapy became dominated by nivolumab when $68 \%$ of the sample was PD-L1 positive and the effectiveness of nivolumab and combination therapy was equal at 4.42 PFQALYs (Figure 2B).

Because the duration and intensity of the AEs differed among the 3 treatment choices, their utility values may have affected the outcome of the CEA. When utility values were changed to reflect only values for stable (0.80) and progressive disease/ death (0.26) without any adjustments for irAEs, ipilimumab continued to be dominated by nivolumab, and combination therapy had an ICER of $\$ 18,947$ per PFQALY when compared with ipilimumab. Compared with nivolumab, combination therapy had an additional cost of $\$ 275,544$ per PFQALY, which still exceeded the $\$ 100,000$ per PFQALY WTP threshold, so nivolumab remained the optimal treatment (Table 3).

In assessing only the cost variables, the ICER was most sensitive to the total drug cost of ipilimumab, although the optimal treatment decision of nivolumab did not change. Thus, we varied the drug cost of ipilimumab from $\$ 1$ to $\$ 132,649$ (base case value). Ipilimumab was no longer dominated by nivolumab when the total drug cost of ipilimumab (4 doses) decreased to approximately $\$ 88,000$. However, nivolumab remained costeffective compared with ipilimumab with an ICER of $\$ 59$ per PFQALY, while combination therapy had an ICER of $\$ 114,858$ per PFQALY compared with nivolumab monotherapy (Table 3). Combination therapy became a viable treatment option (ICER of combination therapy compared with nivolumab was less than the WTP threshold of $\$ 100,000$ per PFQALY) when the total drug cost of ipilimumab was approximately 
$\$ 86,272$. Combination therapy became the optimal treatment dominating nivolumab when the total drug cost of ipilimumab was approximately $\$ 73,520$ (Table 3 ).

\section{Discussion}

Based on our CEA and a WTP of $\$ 100,000$ per PFQALY, nivolumab monotherapy is cost-effective compared with ipilimumab monotherapy and combination therapy for patients as first-line treatment for unresectable or metastatic melanoma, regardless of PD-Ll status. Furthermore, if a patient has a $\mathrm{PD}-\mathrm{L} 1$ negative status, combination therapy is not cost-effective at a WTP of $\$ 100,000$ per PFQALY; however, combination therapy could still be considered as a treatment option when compared only with ipilimumab or compared with nivolumab if $100 \%$ of patients are PD-Ll negative. Also, combination therapy could be cost-effective if decision makers are willing to pay a higher maximum cost per QALY saved. There is some discussion that the WTP especially for anticancer drugs should be increased to at least $\$ 160,000$ per QALYs, and some have suggested increasing this to as high as $\$ 300,000$ per QALY. ${ }^{28}$ However, our analysis shows that, even at this higher WTP threshold, combination therapy would not be cost-effective compared with nivolumab. In addition, because of the lack of consensus in interpreting PD-L1 tests for metastatic melanoma and varying thresholds of PD-Ll expression for a positive test, we cannot confidently recommend combination therapy as a cost-effective choice compared with nivolumab for PD-L1 negative patients at this time.

This result is similar to the findings of the CEAs completed in Australia, which resulted in an ICER of $\$ 44,867$ per QALYS when comparing combination therapy with ipilimumab, resulting in the same cost-effectiveness decision as we did for this comparison..$^{23,24}$ The Australian studies did not compare combination therapy with nivolumab. ${ }^{23}$ Leaving out ipilimumab in our model, however, demonstrated that combination therapy also was not cost-effective compared with nivolumab monotherapy, with an ICER of $\$ 454,092$ per PFQALY gained. Nivolumab is a better comparator with combination therapy, since it is the next least costly alternative.

In the sensitivity analyses, our model was robust to all variables, since there were no changes in the preferred treatment when parameters for all inputs were varied across a plausible range. Nivolumab remained more cost-effective than combination therapy because combination therapy consistently had a higher ICER than the $\$ 100,000$ WTP threshold, and ipilimumab was consistently dominated by nivolumab.

Our sensitivity analyses demonstrated that multiple factors prevented combination therapy from being cost-effective against nivolumab. Primarily, the overall cost of combination therapy is naturally more expensive than either nivolumab or ipilimumab alone because of the combination of 2 drugs and lengthier treatment duration. Although our model showed that immunotherapies achieve remarkable improvements in survival and response, the health state utility of the treatment AEs may still be a huge burden for patients, even under the most favorable assumptions. During the Checkmate-067 trial, $36 \%$ of patients in the combination arm discontinued treatment because of treatment-related AEs, compared with $8 \%$ in the nivolumab arm and $15 \%$ in the ipilimumab arm. ${ }^{12}$ While the higher discontinuation rate for combination therapy may suggest that there is a potential reduction in the overall treatment cost, Checkmate-067 also reported patients on the combination treatment who were administered a median of 4 nivolumab doses and 4 ipilimumab doses. ${ }^{12}$ Therefore, patients who discontinued combination therapy likely accounted for a majority of treatment cost relatively early in their courses. In addition, altering AE utilities did not change the recommendation for nivolumab monotherapy.

Much has been written about value-based pricing and the unsustainable, rising prices of cancer drugs. ${ }^{43,44}$ The innovative nature of the therapies studied here, specifically combination therapy and its effect on lengthening PFS and increasing response rate, must be acknowledged by a higher price. However, the utility may not be high enough to offset the current price set by the manufacturer in the United States.

The use of ipilimumab monotherapy has already been reassessed in the newest NCCN Guidelines (version 3.2016), which now recommend ipilimumab monotherapy solely for second-line or subsequent therapy. ${ }^{19}$ While the price of these immunotherapies will most likely not be reevaluated, health plans must be aware of their benefits and toxicities when examining their value, safety, and affordability.

Ipilumumab and nivolumab are produced and marketed by the same manufacturer. Although nivolumab is priced much lower than ipilumumab, combination therapy would allow the continued presence of both drugs on the market, even as ipilimumab is moved to second-line or subsequent therapy. Similar drugs from other manufacturers (e.g., atezolizumab [Tecentriq]) are in development, and subsequent market pressures for the price of these drugs may decrease their prices.

We also considered the effect of targeting treatment to PD-Ll status of a patient in the ICER analysis. ${ }^{25}$ Emerging evidence indicates that those who are $\mathrm{PD}-\mathrm{Ll}$ positive may respond more favorably to combination therapy compared with PD-L1 negative patients. ${ }^{25,45}$ If this is the case, combination therapy may be considered as a targeted treatment for metastatic melanoma patients with PD-Ll positive tumors. However, since no standardized method for assessing PD-Ll expression exists and the threshold to define PD-Ll status as positive differs between clinical trials (e.g., at least 5\% of tumor cells showing PD-Ll staining for combination therapy or nivolumab trials versus $1 \%$ for pembrolizumab trials), it may be premature to target melanoma treatment based on PD-L1 status. ${ }^{12,14}$ Our sensitivity analysis demonstrated that in a population of $100 \%$ 
PD-L1 negative patients, combination therapy, despite having a lower ICER than the base case, was still not cost-effective (ICER $=\$ 295,903$ per PFQALY) compared with nivolumab and ipilimumab monotherapies at WTP of $\$ 100,000$ per PFQALY.

The biomarker testing process could affect treatment decisions and patient access to anti-PD-Ll therapies. First, it is not clear if detection of tumor cells truly characterizes PD-L1 positive samples. Second, effectiveness and clinical outcomes between different investigational drugs may not be compared, since different companion diagnostic assays with independent definitions of PD-Ll positivity are used. ${ }^{46}$ Clinical trials and any future CEAs that compare new treatments, such as pembrolizumab with combination therapy or nivolumab monotherapy, need to take into consideration the staining thresholds and that PD-Ll assays are not currently interchangeable.

\section{Limitations}

Our model had several limitations. First, the Markov model required several assumptions. For example, when microcosting AEs, we estimated the frequency of follow-up visits, frequency of diagnostic and monitoring labs, and duration of the steroid taper. These assumptions, however, were based on the clinical protocol for the CheckMate-067 trial, combined with clinical knowledge, treatment guidelines, and corroboration from a physician who is an expert in the treatment and management of melanoma, all of which gave us confidence in their accuracy. Efficacy and AE data were taken directly from Checkmate-067, and while a deterministic Markov model is based on the evolution of a hypothetical cohort of patients, the PSA simulates the evolution of real, individual patients. In addition, we varied costs of treating and managing AEs by $\pm 25 \%$ to account for uncertainty, but this did not change our decision.

Furthermore, we used PFS as our only outcome, since OS was not yet available at the time of the analysis. However, even when PFS is weakly correlated with OS, PFS provides a useful indicator of the quality of a drug product to the manufacturer and to practicing physicians. ${ }^{43}$ In using PFS as our outcome, we assumed that OS would follow the PFS Kaplan Meier curve. Other methods for estimating the relationship between PFS and OS include assuming that an incremental benefit in PFS for treatments A and B leads to a proportional gain in OS for each treatment, or one could model independent curves for PFS and OS for each treatment. However, both options have weaknesses, and this study, as with any study based on PFS outcomes, should be followed up with further studies when OS is available. ${ }^{47}$

Since the use of PFS will likely underestimate the life-years gained, we mitigated this by including PFS in the sensitivity analysis, assigning a final utility to the progression/death health state, and dividing that utility in half to adjust for those who died. Moreover, we varied the utility of the progression/ death health state by $\pm 25 \%$, which still resulted in nivolumab monotherapy dominating the other treatments.
We also used ICD-9-CM codes and utility values for AEs that were not always exactly correlated to irAEs, since direct results for general health status and quality of life were not published at the time of the analysis. This situation is not uncommon in CEAs, so the ICD-9-CM codes were closely matched to the description of the irAE being treated, and utility values were carefully selected based on the closest representation of the published literature. ${ }^{37-42}$ Also, when we ran the model without utility, our results did not change.

Finally, this analysis focused on the cost-effectiveness of combination therapy, nivolumab, and ipilimumab as first-line therapies; the cost associated with the progression/death health state did not include the cost of any second- or third-line treatments for metastatic melanoma, since these data were not available and would change the nature of our question. This study was initiated when the NCCN Guidelines still recommended ipilimumab, nivolumab, and pembrolizumab as first-line treatment options. Pembrolizumab was not included as a comparator in this analysis because of the differences in the clinical trial study designs and patient populations of our comparators. Considering the updated versions of the NCCN Guidelines, pembrolizumab should ideally become one of the comparators of any future cost-effectiveness studies for melanoma.

\section{Conclusions}

This analysis, which demonstrated that nivolumab is more cost-effective than nivolumab-ipilimumab combination therapy and ipilimumab monotherapy, contributes to the discussion on value-based and indication-specific pricing in the oncology space, and has implications for formulary and reimbursement decisions, as well as treatment decisions by physicians and patients. Insurers, health systems, and physicians must consider these results in the context of patient outcomes and efficient care. Future research should include updating the decision tree and Markov model to reflect OS data once they are available, which would more accurately make the comparison between combination therapy and nivolumab and ipilimumab monotherapies and allow for more informed costeffective decisions to be made regarding their use. Despite the weakness of using PFS as an outcome in this CEA, our results are supported by the only other CEA analyses for the comparison they had in common.

We found that combination therapy is not cost-effective using PFS, compared with nivolumab, but is cost-effective compared with ipilimumab. One could still consider the use of combination treatments until OS data become available to provide better long-term outcomes. In addition, future clinical trials and CEAs should compare pembrolizumab to nivolumab and combination therapy according to the updated NCCN Guideline recommendations. Furthermore, CEAs of nivolumab and combination therapy in other disease states is warranted, since these therapies may have broader clinical uses and varying effectiveness. 


\section{Cost-Effectiveness of Nivolumab-Ipilimumab Combination Therapy Compared with Monotherapy for First-Line Treatment of Metastatic Melanoma in the United States}

Since the AWP of nivolumab and ipilimumab may change because of more competition and possible FDA approval of additional indications for nivolumab and combination therapy, further work is needed to understand the clinical benefits and value of these therapies. In the meantime, this work can support the information in the dossier of a health plan when examining the value of formulary recommendation of these combinations or monotherapies as suggested by the AMCP Format for Formulary Submissions.

\section{Authors}

ANNA OH, MSN, MPH, RN, Department of Social and Behavioral Sciences, School of Nursing, University of California, San Francisco. DANG M. TRAN, BS; LEANN C. MCDOWELL, PharmD; DOR KEYVANI, PharmD; JAY ANDREW BARCELON, BS; OSCAR MERINO, BS; and LESLIE WILSON, PhD, Department of Clinical Pharmacy, University of California, San Francisco.

AUTHOR CORRESPONDENCE: Anna Oh, MSN, MPH, RN, 3333 California St., San Francisco, CA 94118. Tel.: 415.502.5092; E-mail:anna.oh@ucsf.edu.
6. Palathinkal DM, Sharma TR, Koon HB, Bordeaux JS. Current systemic therapies for melanoma. Dermatol Surg. 2014;40(9):948-63.

7. Hodi FS, O'Day SJ, McDermott DF, et al. Improved survival with ipilimumab in patients with metastatic melanoma. N Engl J Med. 2010;363(8):711-23.

8. Schadendorf D, Hodi FS, Robert C, et al. Pooled analysis of long-term survival data from phase II and phase III trials of ipilimumab in unresectable or metastatic melanoma. J Clin Oncol. 2015;33(17):188994.

9. Mekinist (trametinib) tablets, for oral use. Novartis Pharmaceuticals Corporation. Revised February 2017. Available at: https://www.pharma us.novartis.com/sites/www.pharma.us.novartis.com/files/mekinist.pdf. Accessed April 21, 2017.

10. Zelboraf (vemurafenib) tablet for oral use. Genentech. Revised April 2017. Available at: http://www.gene.com/download/pdf/zelboraf_prescribing. pdf. Accessed April 21, 2017

11. Tafinlar (dabrafenib) capsules, for oral use. Novartis Pharmaceuticals Corporation. Revised June 2016. Available at: https://www.pharma. us.novartis.com/sites/www.pharma.us.novartis.com/files/tafinlar.pdf. Accessed April 21, 2017.

12. Larkin J, Chiarion-Sileni V, Gonzalez R, et al. Combined nivolumab and ipilimumab or monotherapy in untreated melanoma. N Engl J Med. 2015;373(1):23-34.

13. Robert C, Long GV, Brady B, et al. Nivolumab in previously untreated melanoma without BRAF mutation. N Engl J Med. 2015;372(4):320-30

14. Robert C, Schachter J, Long GV, et al. Pembrolizumab versus ipilimumab in advanced melanoma. N Engl J Med. 2015;372(26):2521-32.

15. National Comprehensive Cancer Network. Melanoma (version 3.2015). Available at: http://www.nccn.org/professionals/physician_gls/f_guidelines. asp. Accessed April 21, 2017

16. U.S. Food and Drug Administration. Approved drugs. Nivolumab in combination with ipilimumab. 2015. Available at: https://wayback.archive-it. org/7993/20170111231627/http://www.fda.gov/Drugs/InformationOnDrugs/ ApprovedDrugs/ucm465274.htm. Accessed April 21, 2017.

17. Postow MA, Chesney J, Pavlick AC, et al. Nivolumab and ipilimumab versus ipilimumab in untreated melanoma. N Engl J Med. 2015;372(21):2006-17.

18. National Comprehensive Cancer Network. Melanoma (version 2.2016). Available at: http://www.nccn.org/professionals/physician_gls/f_guidelines. asp. Accessed April 21, 2017

19. National Comprehensive Cancer Network. Melanoma (version 3.2016). http://www.nccn.org/professionals/physician_gls/f_guidelines.asp. Accessed April 21, 2017.

20. Naidoo J, Page DB, Li BT, et al. Toxicities of the anti-PD-1 and antiPD-Ll immune checkpoint antibodies. Ann Oncol. 2015;26(12):2375-91

21. Neumann PJ. Evidence-based and value-based formulary guidelines. Health Aff (Millwood). 2004;23(1):124-34

22. Academy of Managed Care Pharmacy. The AMCP format for formulary submissions. Version 4.0. April 2016. Available at: http://www.amcp.org/ FormatV4/. Accessed April 21, 2017

23. Bohensky MA, Kim H, Gorelik A, Liew D. Cost-effectiveness of nivolum$\mathrm{ab}$ in combination with ipilimumab in patients with unresectable advanced melanoma in Australia. Ann Oncol. 2015;26(Suppl 9):ix103 [Abstract 344O]. Available at: https://academic.oup.com/annonc/article/26/suppl_9/ ix103/2800974/3440Cost-effectiveness-of-nivolumab-in-combination. Accessed April 21, 2017.

24. Bohensky MA, Pasupathi K, Gorelik A, Kim H, Harrison JP, Liew D. Cost-effectiveness of nivolumab versus ipilimumab in patients with RAF mutation negative unresectable advanced melanoma (BRAF-AM) in Australia. Pigment Cell Melanoma Res. 2015;28(6):753-826.

25. Swaika A, Hammond WA, Joseph RW. Current state of anti-PD-Ll and anti-PD-1 agents in cancer therapy. Mol Immunol. 2015;67(2 Pt A):4-17. 
26. International Federation of Health Plans. 2015 comparative price report: variation in medical and hospital prices by country. July 19, 2016. Available at: http://staticl.squarespace.com/static/518a3cfee4b0a77d03a62c98/t/57d 3ca9529687fla257e9e26/1473497751062/2015+Comparative+Price+Repo rt+09.09.16.pdf. Accessed April 21, 2017.

27. Gold MR, Siegel JE, Russell LB, Weinstein MC. Cost-Effectiveness in Health and Medicine. New York: Oxford University Press; 1996.

28. Neumann PJ, Cohen JT, Weinstein MC. Updating cost-effectiveness-the curious resilience of the \$50,000-per-QALY threshold. N Engl J Med. 2014:371(9):796-97.

29. Briggs AH, Claxton K, Sculpher MJ. Decision Modelling for Health Economic Evaluation. New York: Oxford University Press; 2006.

30. Eddy DM, Hollingworth W, Caro JJ, et al. Model transparency and validation: a report of the ISPOR-SMDM Modeling Good Research Practices Task Force-7. Value Health. 2012;15(6):843-50.

31. Husereau D, Drummond M, Petrou S, et al. Consolidated Health Economic Evaluation Reporting Standards (CHEERS) — explanation and elaboration: a report of the ISPOR Health Economic Evaluation Publication Guidelines Good Reporting Practices Task Force. Value Health. 2013;16(2):231-50.

32. Begg CB, Weinstein MC, Braun P, Doubilet P. Probabilistic sensitivity analysis using Monte Carlo simulation. A practical approach. Med Decis Making. 1985;5(2):157-77.

33. Shao J, Zhong B. Last observation carry-forward and last observation analysis. Statist Med. 2003;22(15):2429-41.

34. U.S. Congressional Budget Office. Prescription drug pricing in the private sector. Pub. No. 2703. January 2007. Available at: https://pdfs.semanticscholar.org/0e4f/3b6aabb2f8d7dfc8f4ld26ac6216ab7daf90.pdf. Accessed April 21, 2017.

35. TheAverageBody.com. Average weight. 2014. Available at: http://www. theaveragebody.com/average_weight.php. Accessed April 21, 2017.

36. Friedman CF, Proverbs-Singh TA, Postow MA. Treatment of the immune-related adverse effects of immune checkpoint inhibitors. JAMA Oncol. 2016;2(10):1346-48.
37. Beusterien KM, Szabo SM, Kotapati S, et al. Societal preference values for advanced melanoma health states in the United Kingdom and Australia. Br J Cancer. 2009;101(3):387-89.

38. Lloyd A, Nafees B, Narewska J, Dewilde S, Watkins J. Health state utilities for metastatic breast cancer. Br J Cancer. 2006;95(6):683-90.

39. Swinburn P, Wang J, Chandiwana D, Mansoor W, Lloyd A. Elicitation of health state utilities in neuroendocrine tumours. J Med Econ. 2012;15(4):681-87.

40. Dobler CC, Martin A, Marks GB. Benefit of treatment of latent tuberculosis infection in individual patients. Eur Respir J. 2015;46(5):1397-406.

41. Chen SC, Bayoumi AM, Soon SL, et al. A catalog of dermatology utilities: a measure of the burden of skin diseases. J Investig Dermatol Symp Proc. 2004;9(2):160-68.

42. Nolan JP, Tarsa NJ, Dibenedetto G. Case-finding for unsuspected thyroid disease: costs and health benefits. Am J Clin Pathol. 1985;83(3):346-55.

43. Howard DH, Bach PB, Berndt ER, Conti RM. Pricing in the market for anticancer drugs. J Econ Perspect. 2015;29(1):139-62.

44. Experts in Chronic Myeloid Leukemia. The price of drugs for chronic myeloid leukemia (CML) is a reflection of the unsustainable prices of cancer drugs: from the perspective of a large group of CML experts. Blood. 2013;121(22):4439-42.

45. Wolchok JD, Kluger H, Callahan MK, et al. Nivolumab plus ipilimumab in advanced melanoma. $N$ Engl J Med. 2013;369(2):122-33.

46. Hansen AR, Siu LL. PD-L1 expression as a predictive biomarker. JAMA Oncol. 2016;2(1):15-16.

47. Davis S, Tappenden P, Cantrell A. A review of studies examining the relationship between progression-free survival and overall survival in advanced or metastatic cancer. August 2012. Available at: http://www.nicedsu.org.uk/PFSOS\%20Report.FINAL.06.08.12.pdf. Accessed April 21, 2017.

48. Guy GP Jr, Ekwueme DU, Tangka FK, Richardson LC. Melanoma treatment costs: a systematic review of the literature, 1990-2011. Am J Prev Med. 2012;43(5):537-45. 
Cost-Effectiveness of Nivolumab-Ipilimumab Combination Therapy Compared with Monotherapy for First-Line Treatment of Metastatic Melanoma in the United States

APPENDIX A Progression-Free Survival Curves and Derived Transition Probabilities: Proportion of Patients from CheckMate-067 Experiencing irAEs and Utility Estimate by Organ System

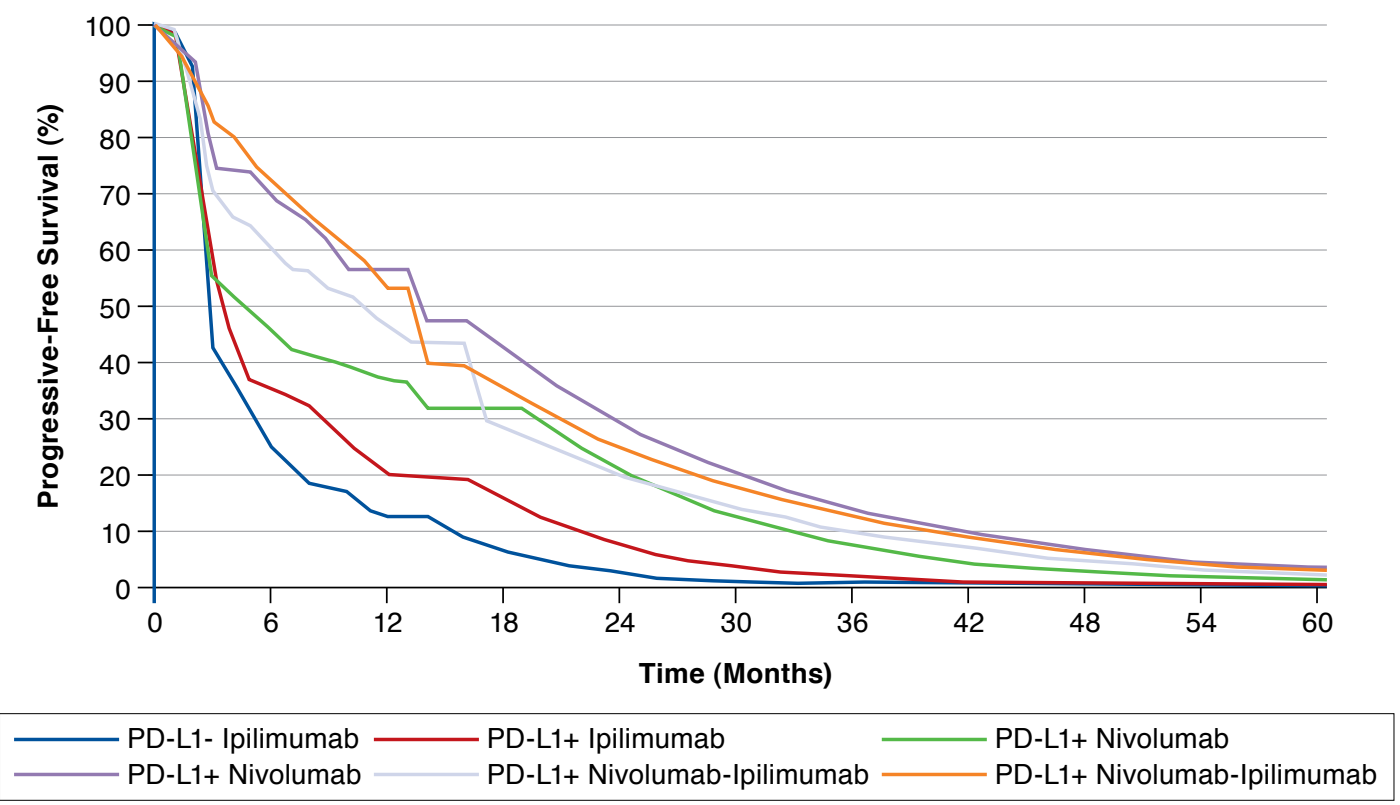

irAE = immune-related adverse event; $P D-L 1=$ programmed death-ligand 1 .

APPENDIX B Tornado Diagram Summarizing One-Way Sensitivity Analyses Within the 95\% Cls

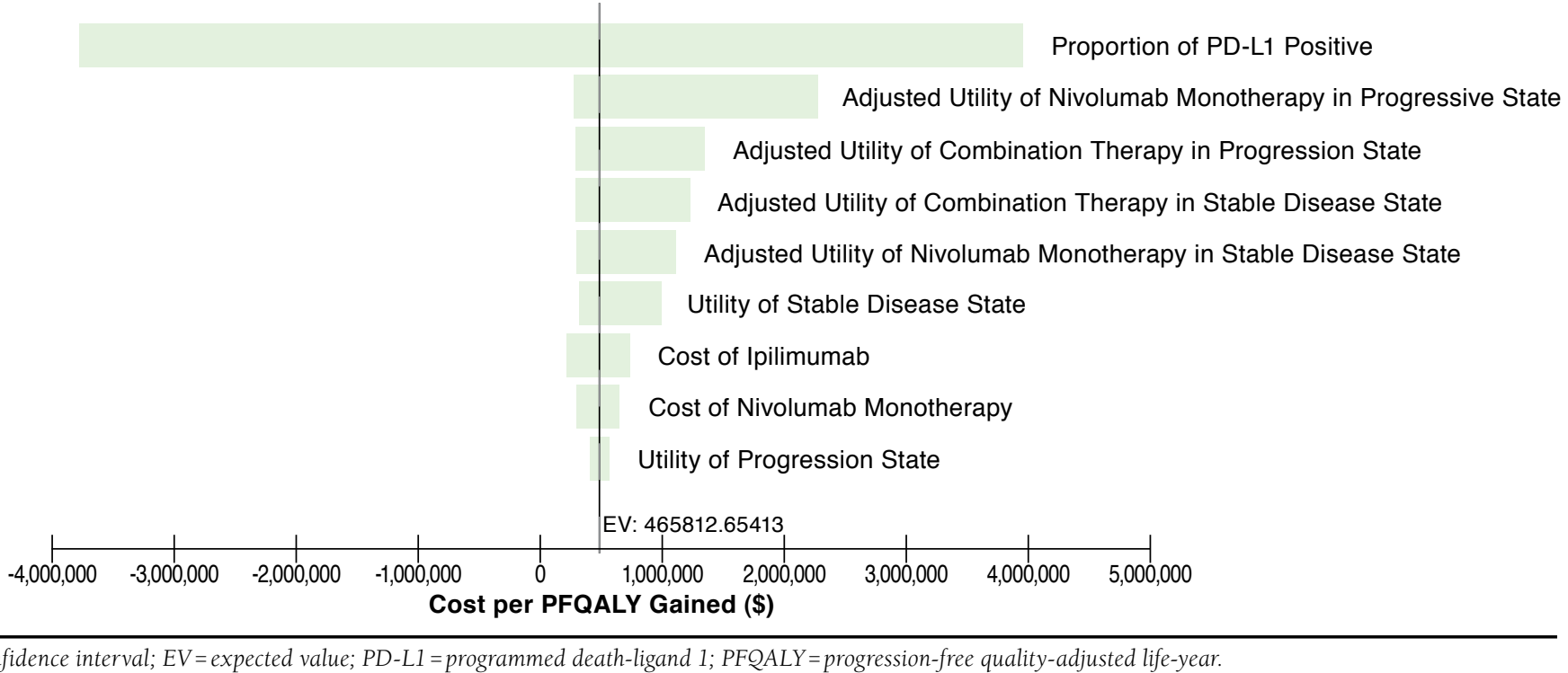

